Meta

Journal des traducteurs

Translators' Journal

\title{
Terminographie juridique et spécificités textuelles
}

\section{Maria da Graça Krieger}

Volume 47, numéro 2, juin 2002

Traduction et terminologie juridiques

URI : https://id.erudit.org/iderudit/008012ar

DOI : https://doi.org/10.7202/008012ar

Aller au sommaire du numéro

Éditeur(s)

Les Presses de l'Université de Montréal

ISSN

0026-0452 (imprimé)

1492-1421 (numérique)

Découvrir la revue

Citer cet article

da Graça Krieger, M. (2002). Terminographie juridique et spécificités textuelles. Meta, 47(2), 233-243. https://doi.org/10.7202/008012ar

\section{Résumé de l'article}

Cet article décrit quelques propositions théorico-méthodologiques adoptées lors de l'élaboration d'un Glossaire multilingue du droit international de l'environnement. Les résultats présentés sont partis du principe selon lequel la prise en compte des spécificités textuelles, discursives et pragmatiques de chaque domaine de connaissance offre des critères pour l'identification de la terminologie étudiée, ainsi que des directives qui permettent de dépasser nombre d'impasses des entreprises terminographiques. On remarque aussi que le progrès de la terminologie est associé à la description du fonctionnement complexe du langage.
Ce document est protégé par la loi sur le droit d'auteur. L'utilisation des services d'Érudit (y compris la reproduction) est assujettie à sa politique d'utilisation que vous pouvez consulter en ligne.

https://apropos.erudit.org/fr/usagers/politique-dutilisation/ 


\title{
Terminographie juridique et spécificités textuelles
}

\author{
MARIA DA GRAÇA KRIEGER \\ Université fédérale du Rio Grande do Sul, Porto Alegre, Brésil
}

\begin{abstract}
RÉSUMÉ
Cet article décrit quelques propositions théorico-méthodologiques adoptées lors de l'élaboration d'un Clossaire multilingue du droit international de l'environnement. Les résultats présentés sont partis du principe selon lequel la prise en compte des spécificités textuelles, discursives et pragmatiques de chaque domaine de connaissance offre des critères pour l'identification de la terminologie étudiée, ainsi que des directives qui permettent de dépasser nombre d'impasses des entreprises terminographiques. On remarque aussi que le progrès de la terminologie est associé à la description du fonctionnement complexe du langage.
\end{abstract}

\begin{abstract}
This article describes some theoretical and methodological propositions adopted in the elaboration of a Multilingual Glossary of Environmental Law. The results show that the identification of the terminology in focus and the guidelines to solve terminological problems can be determined by the textual, discursive and pragmatic specificities of each field of knowledge. This study suggests that progress in the field of terminology is linked to the description of how language is used in different areas.
\end{abstract}

\section{MOTS-CLÉS/KEYWORDS}

Droit International de l'Environnement, glossaire, spécificités textuelles, terminographie juridique

\section{Préliminaires: nouvelles approches terminologiques}

Comme nous le savons, en terminographie le schéma opérationnel ne peut être établi entièrement a priori, étant donné que chaque projet doit répondre à des besoins spécifiques, à des buts sociaux déterminés. Cela requiert la définition d'un ensemble d'aspects pragmatiques concernant la production d'un ouvrage de référence. Ainsi, toute consécution d'un travail terminographique représente le défi d'établir un schéma théorico-méthodologique ponctuel afin de dépasser les différentes étapes de la tâche projetée et d'élaborer un produit qui soit utile à l'usager visé.

Cependant, de même qu'il faut déterminer le schéma structural de l'ouvrage à la lumière de la fonctionnalité voulue, il faut également prendre en compte les spécificités des textes qui matérialisent les communications spécialisées, aussi bien pour procéder à une meilleure identification du répertoire terminologique du domaine que pour établir le schéma informatif général. L'adoption de ce critère, comme condition d'orientation des productions terminographiques, équivaut à inscrire la recherche terminographique dans le cadre épistémologique actuel de la discipline, laquelle abandonne de plus en plus le caractère normatif de ses débuts en faveur d'un paradigme descriptif, ce qui permet d'établir une étape nouvelle et prometteuse de sa trajectoire. 
Dans son parcours actuel, la terminologie est en train de dépasser la phase du privilège du domaine de connaissance, autrement dit celle où la dimension conceptuelle du domaine spécialisé, dans sa virtualité, était considérée comme le seul contexte susceptible d'expliquer l'apparition de ses termes. Il s'agissait d'un temps où dominait «le fondement traditionnel de la terminologie, fondé sur des conceptions ontologiques antérieures à la linguistique saussurienne, selon lesquelles le signe linguistique devient presque invisible et donc éliminable.» Lara (1999: 41).

Aujourd'hui, selon les nouveaux courants terminologiques, les termes ne sont plus étudiés d'un point de vue exclusivement cognitif, mais également linguistique et communicationnel. Nombreux sont les travaux qui développent cette nouvelle approche de la terminologie: les réflexions pionnières d'Alain Rey (1979), suivis de quelques théoriciens comme Sager (1990), Gambier (1991), Gaudin (1993), Slodzian (1994), Temermann (2000). À ce groupe s'ajoutent les propositions de Maria Teresa Cabré et de ses collaborateurs, qui ont été les premiers à formuler, de façon systématique, la critique de la classique Théorie générale de la terminologie, en présentant les bases de la Théorie communicative de la terminologie (1999).

De ces contributions, il résulte une importante avancée dans la compréhension du phénomène terminologique au niveau de ses aspects les plus divers, telles que la variation et la synonymie. Cela montre que le terme, à la différence des croyances antérieures, est une unité linguistique qui se conduit de manière semblable aux unités du lexique général en ce qui concerne les processus de production de signification.

D’un autre côté, les configurations morphologiques des unités lexicales spécialisées des champs de savoir et d'innovations technologiques les plus distinctes montrent qu'elles ressemblent, voire se confondent, aux mots de la langue générale, à la différence des nomenclatures technico-scientifiques classiques, constituées de formants grecs et latins. Les termes ne sont donc pas des étiquettes et ils révèlent leur appartenance aux systèmes linguistiques de plusieurs manières, soit par la consonance aux modèles morphosyntaxiques des langues qui les véhiculent, qu'ils soient originels ou correspondant à des structures néologiques, soit de par leur comportement dans les contextes discursifs.

Dans un même temps, les recherches terminologiques démontrent désormais que les termes sont dynamiques, qu'ils n'appartiennent pas exclusivement à un domaine, mais y prennent une signification spécifique. Cela signifie que le statut d'une unité terminologique définit sa pertinence thématique face à un champ du savoir donné par les décors communicatifs où il s’inscrit. De cette façon, la croyance dans l'idéal de l'exclusivité dénominative et dans la monosémie ne trouve plus de support, de même qu'il n'est plus possible d'établir des frontières rigides entre le lexique spécialisé et le lexique général.

En face du comportement systémique des unités lexicales spécialisées, le travail d'identification terminologique devient beaucoup plus complexe. Comme nous l'avons postulé, la tâche d'identification d'un répertoire terminologique requiert désormais une série de considérations sur le fonctionnement du langage, étant donné que le statut terminologique d'une unité lexicale se lie fortement à des particularités des contextes discursifs où l'unité s'insère. Cela équivaut à reconnaître le rôle des spécificités des univers de discours sur la constitution de leurs terminologies (Krieger 2000: 224). Parmi ces spécificités, on trouve la typologie textuelle elle-même et les buts qui président à la constitution des champs du savoir. 
Le droit est exemplaire pour illustrer ce positionnement, car il ne s'agit pas seulement d'un domaine de connaissance; il est plutôt articulé par des objectifs pragmatiques en raison de l'aménagement juridico-social qu'il établit. À ces aménagements se rattache sa nature déontique primordiale, ce qui explique la présence d'une série de mécanismes linguistiques pragmatiques et sémiotiques qui génèrent des effets d'impérativité dans les textes légaux. En plus, ces mécanismes contribuent à l'expression de l'univers conceptuel du domaine, ainsi qu'à celle d'opérations juridiques nécessaires à l'accomplissement de dispositions légales spécifiques.

«En effet, le premier but de la communication du droit, un domaine humain, social et normatif, est de prescrire des normes de comportement. C'est pourquoi les critères d'attribution du statut terminologique et de reconnaissance des unités lexicales qui composent sa terminologie different de ceux adoptés dans d'autres domaines de connaissance et d'activité avec des objectifs distincts. Ainsi, c'est dans la communication des normes juridiques que se configure sa spécificité.» (Maciel 2001:26).

Les éléments constitutifs du domaine juridique sont à leur tour intimement liés à la configuration de leur répertoire terminologique, dans lesquels, à la différence des autres domaines où dominent les noms, les verbes atteignent souvent un statut terminologique. Cela s'explique par le rôle des verbes dans l'énonciation performative de la loi (Maciel 2001).

Identifier les spécificités de la communication juridique et établir leur rapport à la terminologie du domaine signifie simultanément reconnaître la contribution à la terminologie que peuvent offrir les théories du texte et du discours, y compris le rôle de la pragmatique et de la sémiotique française, ainsi que leurs apports à la narrativité de tout type de texte pour la description des processus de production de signification (Greimas 1976). Autant de contributions fondamentales à l'explication de la production du phénomène terminologique sur le plan textuel.

À partir du point de vue des rapports entre spécificités textuelles, identification et comportement des termes d'un domaine, nous présentons ici quelques solutions méthodologiques adoptées tout au long du processus d'élaboration d'un Glossaire multilangue de droit environnemental international: portugais, espagnol, français, anglais (Krieger et al. 2001). Le langage juridique présente des particularités qui engendrent une série de problèmes pour un traitement terminologique à des fins terminographiques adéquat. Afin d'illustrer l'orientation méthodologique adoptée, nous allons mettre en lumière les trois items suivants : l'identification des caractéristiques de la terminologie du droit environnemental international, l'organisation macrostructurale proposée et le traitement conceptuel des termes dans la microstructure.

\section{La terminologie du droit environnemental international: identification et catégorisation}

La tâche de reconnaissance des termes du droit environnemental international est partie du principe théorico-méthodologique, déjà mentionné, selon lequel l'identification de la terminologie d'un domaine de connaissance ne peut se limiter à l'organisation thématique reflétée par l'arbre de domaine. Celui-ci est un recours important mais non suffisant, car les spécificités constitutives des domaines portent sur la nature des termes, de même qu'elles expliquent la diversité et la conséquente typologie terminologique qu'un même domaine peut comporter. 
Le droit environnemental international est une branche qui rassemble des principes du droit public international et des objectifs du domaine de l'environnement, en établissant les règles de coopération et les engagements passés en vue de la préservation et de la protection de l'environnement dans un cadre qui dépasse les frontières nationales.

Cette expression juridique peut être conçue comme «une manifestation des règles et principes qui créent des obligations et droits de nature environnementale pour les États, les organisations intergouvernementales et les individus» (Nascimento e Silva 1995: 5).

À l'exemple de n'importe quelle branche appliquée du droit international, la législation environnementale se consolide dans les textes des actes internationaux, qui comprennent les traités, conventions, accords, protocoles et documents voisins. Ces textes sont le lieu privilégié de l'établissement de la coopération internationale, puisqu'ils «ont la vertu de déterminer nettement, ou presque, les droits et les obligations des parties contractantes» (Nascimento e Silva 1995: 8).

Les objectifs majeurs de préservation environnementale à l'échelle mondiale, ainsi que la constitution multidisciplinaire du droit environnemental international, se reflètent sur l'organisation textuelle des actes internationaux. Nous pouvons observer que tout texte de cette nature possède un préambule, où sont explicités les principes et les raisons déterminantes des actions coopératives, ainsi que les objectifs poursuivis. Puis sont établies les règles contractuelles et les conditions de coopération mutuelle, ce qui mène à une définition des devoirs des États-parties, signataires des actes, concernant la gestion environnementale. Par cela, on explicite les différentes responsabilités touchant la protection et la préservation d'objets qui sont désormais juridiquement protégés, tels que la couche d'ozone (Convention de Vienne pour la protection de la couche d'ozone) et les tortues marines (Convention interaméricaine pour la protection et la conservation des tortues marines).

En rapport avec ces propositions, on peut reconnaître la coexistence de typologies terminologiques distinctes dans les documents constituant le corpus. À un premier niveau, on observe deux grandes catégories d'unités lexicales spécialisées dans la composition de la terminologie du droit environnemental international: l'une où les termes sont de nature juridico-environnementale, et l'autre qui comprend les unités de valeur juridico-opérationnelle. Bien que tous les termes soient juridiques, puisque créés ou absorbés par les lois environnementales, cette dénomination catégorielle fut motivée par le contenu sémantique véhiculé par les unités spécialisées qui les intègrent. Dans le premier cas, sont exprimés surtout des concepts de base de l'environnement (désertification, gaz à effet de serre) et des objets sous la tutelle des lois environnementales (ressources marines vivantes, zone de la mer Méditerranée), en plus de procédés et d'objets qui provoquent des dommages à l'environnement (arme nucléaire, aérosol); dans le second cas, les termes se réfèrent essentiellement à des mesures, programmes, stratégies et actions de protection internationale de l'environnement (lutte contre la désertification, approche intégrée), ainsi que des principes importants dans la réglementation de la coopération internationale (souveraineté, juridiction).

Les termes de la catégorie juridico-opérationnelle ont été à leur tour subdivisés en général et spécifique; les termes généraux sont communs à tous les traités, par exemple sauvegarde, et les termes spécifiques sont limités au document où ils apparaissent, tel que programmes d'action nationaux. 
Les unités lexicales, que nous avons convenu d'appeler termes juridiques généraux en fonction de leur nature et fonctionnalité dans l'univers du droit, correspondent à la conception selon laquelle elles sont des «termes d'appartenance juridique principale et se prêtent à une première mise en ordre» Cornu (1990: 70). Les termes de cette nature, toujours selon Cornu (1990), sont des «mots-clés du vocabulaire juridique de base», comprenant le vocabulaire judiciaire, ainsi que celui des principales opérations juridiques qui correspondent aux actes juridiques courants.

En complémentarité, une autre catégorie de termes a été envisagée, à savoir celle qui identifie des institutions, organismes et entités, des dénominations fréquemment utilisées dans les documents. Cela s'explique par le fait que les intentions à visée internationale se produisent, pour la plupart, par l'intermédiaire d'actions d'organismes internationaux tels que l'Organisation maritime internationale, responsable du contrôle de la pollution des mers, l'Organisation météorologique internationale et l'Agence internationale de l'énergie atomique, dont les responsabilités s'inscrivent dans leurs domaines respectifs de compétence.

La reconnaissance de la coexistence de ces catégories terminologiques montre que l'identification des termes n'a pas obéi à une simple organisation thématique, mais qu'elle s'est fondée sur la nature des termes apparaissant dans les textes légaux, lesquels véhiculent des concepts, principes, buts, responsabilités, dispositifs et conditions de gestion environnementale à l'échelle internationale.

À l'étape suivante, après un examen plus ponctuel de l'adéquation des unités lexicales sélectionnées comme des candidats à terme, la nomenclature a été effectivement établie à la lumière des critères de pertinence thématique et pragmatique des unités répertoriées, indépendamment de la catégorie. Si la pertinence thématique est un facteur indiscutable d'insertion du terme dans la nomenclature finale, les critères de pertinence pragmatique ouvrent la possibilité de juger l'adéquation de cette insertion sous la perspective de l'image de compétence cognitive de l'usager visé.

Nous n'avancerons pas ici dans la description de ces derniers critères déterminants du répertoire terminologique du Droit environnemental international, puisqu'il importe de souligner que la typologie terminologique identifiée a révélé une correspondance directe avec les objectifs de l'ordonnancement juridique sur le plan international et en ce qui concerne l'environnement. Celui-ci, dans ses différentes configurations, est l'objet sous la tutelle des lois environnementales, partant du principe qu'il s'agit, selon la conception juridico-environnementale, d'un bien de l'humanité qu'il incombe aux nations de préserver pour les générations futures.

\section{L’organisation macrostructurale}

La structure générale du Glossaire a été définie selon une organisation de nature thématique et non purement alphabétique, sur la base des grands thèmes privilégiés par le Droit environnemental international. De l'ensemble du corpus, il a été dégagé huit réseaux thématiques spécifiques, auxquels on a ajouté un réseau appelé Traités plurithématiques, étant donnée la diversité des sujets que comportent certains actes, en plus de la problématique environnementale. L'ensemble spécifique est ainsi constitué:

1. Ressources naturelles: flore et faune/fleuves et bassins;

2. Espace maritime: pollution/droit de la mer;

3. Espace extra-atmosphérique; 
4. Changements morphoclimatiques;

5. Pollution de l'atmosphère;

6. Énergie nucléaire: proscription et interdiction des armes nucléaires/emplois de l'énergie nucléaire/accidents nucléaires;

7. Interdiction des techniques militaires nocives à l'environnement;

8. Transport des personnes et de marchandises.

La proposition d'organiser thématiquement le Glossaire a été définie à partir d'une considération initiale sur les caractéristiques structurales génériques du droit environnemental international. Cette branche du droit n'apparaît pas comme un code juridique unitaire, mais plutôt comme une manifestation éparse de règles et de principes qui créent des obligations et des droits de nature environnementale au plan international. Cette configuration éparse justifie la préférence donnée à cette organisation «macro», fondée sur les principaux thèmes qui reflètent la préoccupation mondiale quant à l'environnement, et ce indépendamment du temps chronologique et des États-parties engagés dans chaque traité.

$\mathrm{Du}$ point de vue formel, chaque réseau thématique est d'abord présenté avec la liste des traités analogues respectifs, toujours dans chaque langue. Suit la présentation individuelle de chaque document, assortie de la liste de ses propres termes. Il est intéressant d'observer que la présentation des traités comprend une série d'informations telles que: objectifs, lieu et date de la signature, portée géographique (restreinte, sans restriction), nature de l'acte (bi- ou multilatérale), zone d'application, variantes dénominatives, dépositaire, documents reliés, en plus des notes, en général concernant la constitution du document. En voici un exemple:

\section{FR TRAITÉ CONVENTION INTERAMÉRICAINE POUR LA PROTECTION ET LA CONSERVATION DES TORTUES MARINES \\ DOMAINE Ressources naturelles: flore et faune \\ PORTÉE GÉOGRAPHIQUE Restreinte \\ NATURE Multilatérale \\ LIEU/DATE/SIGN. Caracas, 01/12/96}

OBJECTIFS Promouvoir la protection et la conservation ainsi que le rétablissement des populations de tortues marines et des habitats dont elles dépendent, sur la base de données scientifiques les plus fiables qui soient disponibles et compte tenu des caractéristiques environnementales, socio-économiques et culturelles des parties.

NOTES La Convention présente quatre annexes.

ZONE D'APPLICATION Le territoire terrestre de chacune des parties sur le continent américain, ainsi que les zones maritimes de l'océan Pacifique, à l'égard desquelles chacune des parties exerce sa souveraineté, ses droits souverains ou sa juridiction sur les ressources biologiques marines, conformément au droit international tel qu'il est reflété dans la Convention des Nations Unies sur le droit de la mer.

DOCUMENTS RELIÉS Convention des Nations Unies sur le droit de la mer; Conférence des Nations Unies sur l'environnement et le développement; Accord portant création de l'Organisation mondiale du commerce et l'Accord général sur les tarifs douaniers et le commerce.

Ces données ont été considérées par les spécialistes comme étant importantes pour l'usager qui essaie de s'insérer dans le droit des traités. À vrai dire, il n'y a pas de modèle unique préétabli en ce qui concerne la macro- ou même la microstructure des glossaires. Dans ce cas, nous avons réuni des informations de nature encyclopédique avec la terminologie du domaine, en élaborant un produit qui se caractérise par une base informationnelle consistante. Toujours dans le but de compléter le réseau thé- 
matique, à chaque acte fondateur ont été ajoutés les documents produits ultérieurement tels que les ajustements complémentaires et amendements.

Ainsi, chacune des sections, instituée par les affinités thématiques entre les traités, s'est constituée dans un univers conceptuel à la fois spécifique et large, permettant à l'usager une vision globale et en même temps particularisée de l'occurrence du terme. De cette manière, la conceptualisation et le rôle du terme dans le répertoire du domaine sont plus clairs. En résumé, toutes ces spécificités du domaine du droit environnemental international ont été déterminantes pour l'établissement d'une organisation générale thématique, dans le but de rendre la consultation du glossaire la plus productive possible.

D'un autre côté, la proposition thématique a également pris en compte le fait que la plupart des termes des traités environnementaux sont conceptualisés restrictivement, c'est-à-dire que leur sens ainsi que les implications juridiques que comporte chaque unité spécialisée sont circonscrits au cadre du texte où apparaît l'unité. Cela s'observe particulièrement par rapport aux termes juridico-spécifiques, tels que comité consultatif d'experts. Quant aux termes juridico-opérationnels généraux, ils possèdent une conceptualisation identique dans toute la documentation, ce qui s'explique par le fait que cette catégorie correspond surtout à la terminologie générale du droit des traités. Bien que le concept juridique soit réitéré indépendamment de branche et d'occurrence textuelle, les termes de cette catégorie terminologique reçoivent souvent une signification particulière dans les différents actes, étant donnée l'implication juridique que leur usage engendre. Cette situation se produit même avec un terme aussi générique que États-parties.

L'observation de ce comportement des contextes de signification où apparaît le répertoire terminologique recherché a aussi contribué à considérer comme plus pertinente une organisation générale articulée par des réseaux institués sur les grands thèmes environnementaux, plutôt qu'une simple relation alphabétique de la nomenclature générale du domaine. En même temps, cette syntaxe discursive qui parcourt le lexique juridique dans les textes des actes a influencé la conception de la matrice dessinée pour la microstructure de l'ouvrage, comme nous le verrons ci-dessous.

\section{Traitement terminographique : définitions et notes}

La compréhension du corpus en tant que texte et la reconnaissance des particularités de la communication dans le cadre du droit environnemental international ont également orienté des décisions concernant la microstructure du Glossaire. À ce sujet, on peut remarquer le nombre restreint de définitions dans les traités. C'est une caractéristique particulière de ce champ juridique, bien qu'elle ne soit pas limitée à celuici, car on la trouve d'une manière générale dans les textes de législation.

Lorsqu'il y a des définitions, elles apparaissent sous la forme d'un accord explicite sur le concept véhiculé, comme le montre le contexte du terme habitat des tortues marines, emprunté à la Convention interaméricaine pour la protection et la conservation des tortues marines (art. I):

"On comprend par habitat des tortues marines tous les environnements aquatiques et terrestres que les tortues utilisent lors d'un stade quelconque de leur cycle biologique."

À côté des définitions formalisées, il y a aussi des concepts véhiculés informellement, mais à échelle réduite. Selon les spécialistes, le droit public international a 
l'habitude d'éviter au maximum les définitions pour des raisons d'économie dans les échanges entre les parties, c'est-à-dire que la finalisation de tout accord tend à devenir impossible s'il faut s'accorder sur tous les concepts engagés lors des négociations.

En fait, le droit environnemental international ne constitue pas un champ de production de savoir environnemental mais de propositions de coopération internationale, d'établissement des devoirs et des droits des nations sur l'environnement, compris comme un bien de l'humanité à préserver pour les générations futures. Ainsi, plus que la conceptualisation de termes environnementaux, ce sont les implications légales explicitées dans le contexte d'occurrence du terme qui deviennent importantes, autrement dit les déterminations que la loi stipule, avec l'agencement procédural nécessaire pour combattre la dégradation de l'environnement afin d'offrir des conditions pour le préserver.

Ces caractéristiques constitutives du droit environnemental international ont également orienté autant la structuration de la présentation des aspects conceptuels du règlement environnemental international que les implications juridiques manifestées dans les contextes d'occurrence des termes. En réalité, lorsqu'on a l'intention d'organiser un répertoire lexical représentatif d'un domaine, il faut trouver des solutions qui permettent à l'usager de comprendre le rôle d'unités lexicales importantes dans les communications spécialisées, même si elles apparaissent sans définition et souvent dans des contextes discursifs vides d'information sémantique particulière.

Plus spécifiquement, nous avons décidé que le traitement conceptuel et juridique des termes choisis obéirait à deux modèles de base:

- concernant les termes suivis d'énoncé définitoire, cet énoncé formel a été reproduit dans le champ même de la définition, avec l'indication de la source bibliographique et de la localisation - article et paragraphe - dans le texte légal;

- concernant les termes sans définition, mais sur lesquels le texte juridique établit un objectif particulier ou une contrainte légale, cette observation sera consignée dans le champ réservé aux notes.

Avec les notes juridiques, nous avons l'intention d'orienter la consultation, tout en justifiant la pertinence de l'usage du terme à la lumière de son milieu de signification dans le document, ce qui correspond à une manière d'expliquer l'implication légale correspondante. Pour cela, nous avons reproduit intégralement le contexte d'occurrence de l'unité lexicale ou rédigé une synthèse des déterminations légales, suivi de la consignation de la source référentielle informant en détail l'article, le paragraphe, l'incise. Dans les cas d'information élaborée, l'indication référentielle du texte sur lequel la note s'est fondée est précédée de l'abréviation de l'expression confer (Cf.). Cette forme de traitement est illustrée ci-dessous:

MESURES DE PROTECTION $f$ - $f$ [art. 4] NOTES Chaque partie prend les mesures appropriées et nécessaires, conformément au droit international et sur la base des données scientifiques les plus fiables dont elle dispose, en vue de la protection, conservation et rétablissement des populations de tortues marines et de leurs habitats: sur leur territoire terrestre et dans les zones maritimes sur lesquelles elle exerce sa souveraineté, ses droits souverains ou sa juridiction, situés dans la zone d'application de la Convention; sans préjudice de ce qui est établi dans l'article 3, dans les zones de haute mer, à l'égard des navires autorisés à battre son pavillon. (cf. art. 4, 1) (Convention interaméricaine pour la protection et la conservation des tortues marines) 
Un autre exemple de l'utilisation du système de notes adopté, qui comprend outre les notes juridiques - les notes techniques, linguistiques et de traduction, est le terme aérosol, qui n'est pas défini dans le texte; il s'agit d'un terme pris à l'annexe de la Convention de Vienne pour la protection de la couche d'ozone:

\section{Aérosol}

Notes: En raison des effets nocifs exercés par les aérosols sur la couche d'ozone, on a convenu de mener des observations systématiques sur leurs propriétés et distribution depuis le sol jusqu'à la mésosphère, en utilisant des systèmes au sol et des systèmes sur satellite (cf. Annexe, 1, 2, d, vi).

Il faut souligner que parfois les termes ont reçu, en plus des définitions, des notes, lorsque leur utilisation avait une implication juridique conséquente, comme dans le cas d'arme nucléaire (Traité visant l'interdiction des armes nucléaires en Amérique latine):

DÉFINITION Tout dispositif susceptible de libérer de l'énergie nucléaire de manière non contrôlée, et qui possède un ensemble de caractéristiques propres à l'emploi à des fins belliqueuses.

NOTES L'emploi, la fabrication, l'acquisition et la possession de toute arme nucléaire sont interdits sur les territoires soumis à la juridiction des parties contractantes. (cf. art. 1). L'engin pouvant servir au transport ou à la propulsion du dispositif n'est pas compris comme arme nucléaire, s'il peut être séparé du dispositif et ne fait pas partie intégrante de celui-ci. (cf. art. 5)

Toujours en ce qui concerne le système de notes pour les situations d'occurrence de termes qui ne présentent pas de définition, nous soulignons que cette solution, parmi d'autres facteurs, s'est surtout fondée sur le principe de la crédibilité envers l'univers juridique. Il ne nous incombait pas d'élaborer des définitions si le droit, en principe, les refuse ni d'attribuer une définition à un terme, c'est-à-dire d'emprunter une définition à une autre source, telle que la législation brésilienne, en la transposant simplement dans un contexte légal de portée internationale. Et ce parce que, outre l'inadéquation, le produit perdrait en crédibilité si l'on adoptait un critère qui ne respecte pas les spécificités conceptuelles et la validité juridique des dispositions de chaque acte international dans le cadre de l'environnement.

Quoi qu'il en soit, nous avons essayé avec les notes juridiques de contextualiser l'univers de signification de certaines unités lexicales propres au langage juridique. Ce traitement informationnel — définition et notes — permet aussi de comprendre que le langage juridique comporte une distinction, c'est-à-dire la différence entre sens et signification du discours juridique:

Le sens de ses termes est circonscrit, mais leur signification n'est établie que dans une situation donnée, très précise, appliquée à un cas particulier. Autrement dit, le langage du droit n'est jamais (ou presque) achevé, c'est un langage se faisant, en voie de réalisation permanente (Gémar 1991: 282).

\section{Brèves considérations finales}

Les aspects ici décrits, concernant l'élaboration d'un Glossaire multilingue de droit environnemental international, ont d'abord eu pour but de réaffirmer que le schéma opérationnel pour l'élaboration d'un produit terminographique ne peut pas toujours 
être établi a priori. Au contraire, la prise en compte des spécificités textuelles et discursives de chaque domaine de savoir offre des chemins pour l'adoption de solutions méthodologiques qui permettent de dépasser nombre d'impasses des entreprises terminographiques.

Dans le cas du droit environnemental international, l'organisation terminographique par des domaines thématiques a cherché à exprimer la particularité structurale constitutive du domaine, qui n'est pas celle d'un code juridique unitaire, chronologiquement et thématiquement ordonné, malgré l'axe commun: les engagements communs pour la préservation de l'environnement au niveau international. Par là, nous avons essayé de mieux répondre au principe pragmatique de fonctionnalité de l'ouvrage, rendant la consultation terminologique plus contextualisée que lorsque l'on n'offre à l'usager qu'une nomenclature organisée alphabétiquement.

En même temps, le traitement conceptuel adopté pour les termes, c'est-à-dire l'adoption du système de notes afin de rendre compte des implications juridiques liées à l'utilisation des termes - et ce surtout quand ils ne sont pas accompagnés d'une définition - est le résultat du respect de la forme d'expression de cette branche juridique. De cette façon, nous avons cherché à élaborer un Glossaire contenant des informations susceptibles d'éclairer la signification spécifique que l'emploi du terme actualise, ainsi que d'offrir à l'ouvrage la nécessaire fiabilité, étant donnée sa fidélité au texte légal.

$\mathrm{Au}$ cœur de ces propositions terminographiques, qui n'ont pas de valeur de modèle mais seulement de solutions possibles parmi tant d'autres, se détache la fonctionnalité de la reconnaissance de catégories terminologiques distinctes pour l'identification du lexique de signification spécialisée, propre au droit environnemental international. La constitution du répertoire lexical hybride de cette branche juridique a révélé sa compatibilité avec les spécificités constitutives du domaine. Il importe de dire que les catégories terminologiques qui coexistent dans les textes des actes internationaux contribuent à l'expression de l'aspect déontique propre au droit environnemental, ainsi qu'à la circonscription juridique de concepts, d'objets sous tutelle, des instances et mécanismes opérationnels que la loi stipule afin d'établir ses objectifs d'agencement en vue de la préservation de l'environnement. Cela confirme le point de vue selon lequel la constitution d'une terminologie est intimement liée aux spécificités de chaque domaine.

Ainsi, les fondements adoptés dans l'organisation générale du Glossaire de droit environnemental multilangue, ici présenté, permettent de réaffirmer que la prise en compte de la nature constitutive du domaine est un principe théorico-méthodologique de base pour l'identification des unités terminologiques, pour leur traitement, et elle peut aussi offrir des directives pour l'organisation macrostructurale d'un outil terminographique.

Par conséquent, il est également possible de dire que la terminologie est conduite à reconnaître que son progrès est directement lié à l'intégration de composantes de l'ordre de la textualité et de la discursivité dans l'appareil théorico-méthodologique qui la soutient. En accueillant la complexité du fonctionnement du langage et en cherchant à identifier les mécanismes de production des terminologies dans les différents décors communicatifs, la terminologie avancera encore plus dans la compréhension du phénomène terminologique. 


\section{RÉFÉRENCES}

CABRÉ, M. T. (1999) : La terminología: representación y comunicación. Barcelona, IULA.

Cabré, M. T. et M. Lorente (dir.). Terminología y modelo culturales. Barcelona, Pompeu Fabra, p. 39-60.

Convention de Vienne pour la protection de la couche d'ozone (1998): Recueil des Traités. Traités et accords internationaux. v. 1513. New York: United Nations.

Convention interaméricaine pour la protection et la conservation des tortues marines. Disponible à $<$ http://www.seaturtle.orgliac/franc/eng $>$ en novembre 1999.

Cornu, G. ( 1990): Linguistique juridique. Paris, Montchrestien.

GAMBier, Y. (1991): «Travail et vocabulaires spécialisés: prolégomènes à une socioterminologie». Meta, 36-1, p. 8-15.

Gaudin, F. (1993) Pour une socioterminologie. Rouen, Université de Rouen.

GÉmAR, J.-C. (1991) : «Terminologie, langue et discours juridiques. Sens et signification du langage du droit», Meta 36-1, p. 275-283.

Krieger, M. G. (2000) : «Terminologia revisitada ». Delta, v.16, n.2, pp.209-228.

Krieger, M. G., A. M. Maciel, M. J.B. Finatto \& P.C. Ramos (2001): Glossário Multilíngüe de Direito Ambiental Internacional. Porto Alegre, UFRGS.

LARA, L. F. ( 1999) : «Término y cultura: hacia una teoría del termo».

Maciel, A. M. (2000) : Para o reconhecimento da especificidade do termo jurídico, Porto Alegre, UFRGS.

Nascimento E Silva, J. E.: Direito Ambiental Internacional, Rio de Janeiro, Thex, 1995.

ReY, A. (1979): La terminologie: noms et notions, Paris, PUF, 1979.

SAGER, J. C. Curso práctico sobre el procesamiento de la terminología. Fundación Germán Sánchez Ruipérez, Madrid.

SLodzian, M. (1995) : «Comment revisiter la doctrine terminologique aujourd'hui ?» La Banque des Mots 7, Paris, p. 11-18.

Temmerman, R. (2000) Towards New Ways of Terminology Description, John Benjamins, Amsterdam/Philadelphia.

Traité visant l'interdiction des armes nucléaires en Amérique latine. (1968), Recueil des Traités. Traités et accords internationaux, v. 634. New York, United Nations. 